\title{
Acknowledgment to Reviewers of Tourism and Hospitality in 2021
}

\author{
Tourism and Hospitality Editorial Office
}

Citation: Tourism and Hospitality

Editorial Office. Acknowledgment to Reviewers of Tourism and Hospitality in 2021. Tour. Hosp. 2022, 3, 114-115. https://doi.org/10.3390/ tourhosp3010008

Published: 27 January 2022

Publisher's Note: MDPI stays neutral with regard to jurisdictional claims in published maps and institutional affiliations.

Copyright: () 2022 by the author. Licensee MDPI, Basel, Switzerland. This article is an open access article distributed under the terms and conditions of the Creative Commons Attribution (CC BY) license (https://creativecommons.org/licenses/by/4.0/).
MDPI AG, St. Alban-Anlage 66, 4052 Basel, Switzerland

Rigorous peer-reviews are the basis of high-quality academic publishing. Thanks to the great efforts of our reviewers, Tourism and Hospitality was able to maintain its standards for the high quality of its published papers. Thanks to the contribution of our reviewers, in 2021, the median time to first decision was 16 days and the median time to publication was 38 days. The editors would like to extend their gratitude and recognition to the following reviewers for their precious time and dedication, regardless of whether the papers they reviewed were finally published:

\begin{tabular}{|c|c|}
\hline Alves, Juliana Araújo & Gross, Sven \\
\hline Baggio, Jacopo & Hernández, Juan María \\
\hline Barbieri, Carla & Ingaldi, Manuela \\
\hline Bernat, Sebastian & Irimias, Anna \\
\hline Bryła, Paweł & Ishaq, Muhammad Ishtiaq \\
\hline Buer, Christian & Kar, Samarjit \\
\hline Carral Vilariño, Emilio V. & Kardos, Mihaela \\
\hline Cerutti, Stefania & Kastenholz, Elisabeth \\
\hline Chiu, Yi-Bin & Korstanje, Maximiliano \\
\hline Choi, Suh-Hee & Kubátová, Jaroslava \\
\hline Coelho, António & Łapko, Aleksandra \\
\hline Corsale, Andrea & Liasidou, Sotiroula \\
\hline De Almeida, Cláudia Ribeiro & Linnes, Cathrine \\
\hline de Medici, Stefania & Lopes, João M. \\
\hline Diez Bosch, Miriam & Lotko, Aleksander \\
\hline Di-Virgilio, Francesca & Lucas, Ana \\
\hline Drago, Carlo & Malkowski, Arkadiusz \\
\hline Dynowski, Piotr & Marine-Roig, Estela \\
\hline Dziadkowiec, Joanna & Merciu, Cristina \\
\hline Espeso-Molinero, Pilar & Messina, Enza \\
\hline Falk, Martin Thomas & Mirón Sanguino, Ángel Sabino \\
\hline Fan, Daisy & Moral-Moral, María \\
\hline Fernández-Laso, María Cristina & Muresan, Iulia \\
\hline Foris, Diana & Naumov, Nikola \\
\hline Frondizi, Rocco & Nunes, Katia \\
\hline Fusté-Forné, Francesc & O'Connor, Peter \\
\hline Fyall, Alan & Oliveira, Márcio \\
\hline Gallego-Garcia, Sergio & Osorio, Carlos \\
\hline García-Delgado, F. Javier & Panasiuk, Aleksander \\
\hline Gawlik, Agnieszka & Parzych, Krzysztof \\
\hline Giachino, Chiara & Pforr, Christof \\
\hline Gil de Arriba, Carmen & Pranskūnienè, Rasa \\
\hline
\end{tabular}


Raszka, Beata

Relich, Marcin

Rodríguez-López, Nuria

Roy, Hiran

Ruban, Dmitry

Rubira-García, Rainer

Scarpaci, Joseph L.

Sousa, Nuno

Stoian, Claudia Iuliana

Szalonka, Katarzyna

Timothy, Dallen
Tooman, Heli

Trono, Anna

Valeri, Marco

Vuin, Ana

Wu, Kun-Shan

Yoo, Changsok

Zaharova, Olga V.

Zielińska-Szczepkowska, Joanna

Zmyślony, Piotr

Zopiatis, Anastasios 